\title{
Welcome to the New Open Access NeuroSci
}

\author{
Lucilla Parnetti ${ }^{1, *} \mathbb{0}$, Jonathon Reay ${ }^{2}$, Giuseppina Martella ${ }^{3}{ }^{-}$, Rosario Francesco Donato ${ }^{4}(\mathbb{D}$, \\ Maurizio Memo ${ }^{5}$, Ruth Morona ${ }^{6}$, Frank Schubert ${ }^{7}$ and Ana Adan ${ }^{8,9}$ (D) \\ 1 Centro Disturbi della Memoria, Laboratorio di Neurochimica Clinica, Clinica Neurologica, \\ Università di Perugia, 06132 Perugia, Italy \\ 2 Department of Psychology, Teesside University, Victoria, Victoria Rd, Middlesbrough TS3 6DR, UK; \\ J.Reay@tees.ac.uk \\ 3 Laboratory of Neurophysiology and Plasticity, Fondazione Santa Lucia, and University of Rome Tor Vergata, \\ 00143 Rome, Italy; martella@med.uniroma2.it \\ 4 Department of Experimental Medicine, University of Perugia, 06132 Perugia, Italy; rosario.donato@unipg.it \\ 5 Department of Molecular and Translational Medicine, University of Brescia, 25123 Brescia, Italy; \\ maurizio.memo@unibs.it \\ 6 Department of Cell Biology, School of Biology, University Complutense of Madrid, \\ Av. Jose Antonio Novais 12, 28040 Madrid, Spain; r.morona@bio.ucm.es \\ 7 School of Biological Sciences, University of Portsmouth, Hampshire PO1 2DY, UK; frank.schubert@port.ac.uk \\ 8 Department of Clinical Psychology and Psychobiology, University of Barcelona, 08035 Barcelona, Spain; \\ aadan@ub.edu \\ 9 Institute of Neurosciences, University of Barcelona, 08035 Barcelona, Spain \\ * Correspondence: lucilla.parnetti@unipg.it
}

Received: 6 August 2020; Accepted: 17 August 2020; Published: 3 September 2020

check for updates

\section{Message from Editor-in-Chief: Prof. Dr. Lucilla Parnetti}

With sincere satisfaction and pride, I present to you the new journal, NeuroSci, for which I am pleased to serve as editor-in-chief. To date, the world of neurology has been rapidly advancing, NeuroSci is a cross-disciplinary, open-access journal that offers an opportunity for presentation of novel data in the field of neurology and covers a broad spectrum of areas including neuroanatomy, neurophysiology, neuropharmacology, clinical research and clinical trials, molecular and cellular neuroscience, neuropsychology, cognitive and behavioral neuroscience, and computational neuroscience. Members of our editorial board will welcome the contributions in this wide field of neurosciences. The following are welcome messages from some editorial board members:

Dr. Jonathon Reay: The world of psychopharmacology and drug discovery is moving at pace and clinical and pre-clinical human trials are providing ever increasing insight into the factors and personal characteristics that lead to effective prevention, detection, treatment and symptom management of clinical conditions. This cross-disciplinary, open-access journal offers an opportunity for the presentation of novel data in the field of psychopharmacology and welcomes pre-clinical and clinical trials that lead to a further understanding of prevention, detection, treatment and symptom management of clinical conditions. The journal also welcomes review articles in this area.

Dr. Giuseppina Martella: The world of neurophysiology has made a great advance in the past two decades. Thanks to sophisticated imaging techniques, a great part of the intricate anatomical connections of the brain are being defined in detail. We have had the opportunity to integrate the knowledge of brain anatomy with the functioning necessary to understand the neurobiological basis of human neurophysiology and to determine the mechanisms that underlie locomotor functions. The research on specific transgenic animal models has brought out the biochemical, molecular, and genetic mechanisms that determine brain structure and functions in physiological versus pathological conditions. All this knowledge has had a great impact on therapies and management of human patients, suggesting the possibility to prevent and cure many disorders. 
Given its cross-disciplinary character, the open-access journal offers a great opportunity to present novel data in the field of neurophysiology, physiology, neurosciences, neurobiology, neuroendocrinology, behavioral neuroscience, clinical neuroscience, cognitive neuroscience, computational neuroscience, cultural neuroscience, neuro-engineering, neuroimaging, neurogenetics, neuroinformatics, and neurolinguistics. The aim of this journal is to collect original papers, reviews, case reports, and other forms of scientific communication that could attract the interest of scientists and can contribute to better understanding of the huge world of functions and dysfunction of the brain.

Prof. Dr. Rosario Francesco Donato: Neuroscience is a rapidly evolving field thanks to new technologies that allow the analysis of intracerebral connections and functional (sometimes reciprocal) relationships between different cortical areas and between the cerebral cortex and subcortical structures in real time in the living (experimental animal or living subject). Furthermore, the wide range of cellular and molecular biology and neurophysiology techniques available today allows an interdisciplinary approach to the examination of a given experimental problem with potential consequences in the clinical application field. The editorial board of this journal welcomes experimental work and reviews in any field of neuroscience.

Prof. Dr. Maurizio Memo: Digitalisation has significantly changed the dynamics of scientific writing as well as the way to gain and share scientific knowledge. This is opening new avenues for connecting science to scientists and scientists to the real world. The editors will stay aware of these changes and make selection of the coming news being focused on specific themes such as data robustness, collaborative science, new biomedical models, ethical challenges in the digital age, and social impacts.

Prof. Dr. Ruth Morona: Neurosciences encompass a large number of disciplines including morphological, biochemical physiological, clinical and behavioral aspects of the study of the nervous system and neuronal function. This open access journal publishes rigorously peer-reviewed research across a wide spectrum of specialties and disciplines that allow a broad integration of our knowledge in order to design novel strategies in the hottest current areas.

Dr. Frank Schubert: Fascination with the nervous system remains unbroken after thousands of years of research into its anatomy, its physiology, and its cellular and molecular basis. Health conditions like neurodegenerative diseases, dementia, or cancer of the nervous system remain major challenges of the 21st century. NeuroSci provides an exciting new open access platform to bring together fundamental and applied research across the many disciplines of neuroscience.

Prof. Dr. Ana Adan: The world of chronobiology is essential for a comprehensive understanding of multiple scientific disciplines. Since the 1950s, with the development of adequate recording techniques, both basic and applied findings of chronobiology have not ceased. Its implications are found both at the genetic level (clock genes), physiological or neuroendocrine levels as well as in cognitive and behavioral aspects, underlying as explanatory factors the maintenance of health and quality of life in healthy individuals. It provides explanatory elements in numerous physical pathologies, such as cancer and cardiovascular disorders, and mental disorders such as schizophrenia, depression or substance-use disorders. In all cases, consideration of rhythmic expression can provide indisputable benefits in improving preventive management and/or treatment. This open access journal offers an opportunity to present novel data in the field of chronobiology and welcomes pre-clinical or clinical papers that represent an advance in the knowledge of this area.

We all invite you to submit your comprehensive reviews, original research papers and high-quality communications, and to join us in making this new journal a success!

Conflicts of Interest: The authors declare no conflict of interest.

(C) 2020 by the authors. Licensee MDPI, Basel, Switzerland. This article is an open access article distributed under the terms and conditions of the Creative Commons Attribution (CC BY) license (http://creativecommons.org/licenses/by/4.0/). 